Article

\title{
Association between Fruit Consumption and Lipid Profile among Children and Adolescents: A National Cross-Sectional Study in China
}

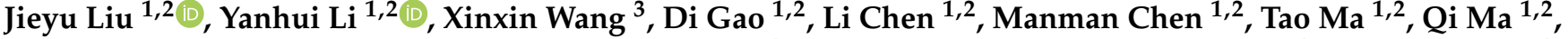

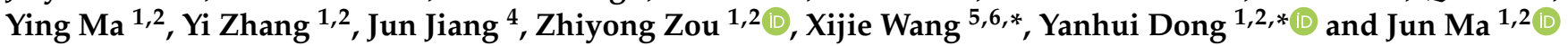

1 Institute of Child and Adolescent Health, School of Public Health, Peking University, Beijing 100191, China; jieyulynne@163.com (J.L.); yanhui_lyh@163.com (Y.L.); gaodi1993@163.com (D.G.); clcl@bjmu.edu.cn (L.C.); 1911210173@pku.edu.cn (M.C.); 1610306216@pku.edu.cn (T.M.); 18702110295@163.com (Q.M.); mypku232@163.com (Y.M.); 1710306140@pku.edu.cn (Y.Z.); harveyzou2002@bjmu.edu.cn (Z.Z.); majunt@bjmu.edu.cn (J.M.)

2 National Health Commission Key Laboratory of Reproductive Health, Peking University, Beijing 100191, China

3 School of Public Health and Management, Ningxia Medical University, Yinchuan 750004, China; wangxinxin291314@163.com

4 Department of Plant Science and Landscape Architecture, University of Maryland, College Park, MD 20742, USA; sherryj@umd.edu

5 Vanke School of Public Health, Tsinghua University, Beijing 100084, China

6 Institute for Healthy China, Tsinghua University, Beijing 100084, China

* Correspondence: xijie_wang@126.com (X.W.); dongyanhui@bjmu.edu.cn (Y.D.); Tel.: +86-10-62782199 (X.W.); +86-10-82801624 (Y.D.); Fax: +86-10-82801178 (Y.D.)

check for

updates

Citation: Liu, J.; Li, Y.; Wang, X.; Gao,

D.; Chen, L.; Chen, M.; Ma, T.; Ma, Q.;

Ma, Y.; Zhang, Y.; et al. Association

between Fruit Consumption and

Lipid Profile among Children and

Adolescents: A National

Cross-Sectional Study in China.

Nutrients 2022, 14, 63. https://

doi.org/10.3390/nu14010063

Academic Editor: Stephen Ives

Received: 5 November 2021

Accepted: 22 December 2021

Published: 24 December 2021

Publisher's Note: MDPI stays neutral with regard to jurisdictional claims in published maps and institutional affiliations.

Copyright: (c) 2021 by the authors Licensee MDPI, Basel, Switzerland. This article is an open access article distributed under the terms and conditions of the Creative Commons Attribution (CC BY) license (https:// creativecommons.org/licenses/by/ $4.0 /)$.

\begin{abstract}
To investigate associations between fruit consumption and lipid profiles, and to further explore a satisfactory level of frequency and daily fruit intake for children and adolescents. A national sample of 14,755 children and adolescents aged 5-19 years from seven provinces in China were recruited. Fasting blood samples were collected to test the lipid profile. Information regarding fruit consumption and other characteristics was collected by questionnaires. Logistic regression models adjusting for confounding covariates were applied to calculate the odds ratio (OR) and 95\% confidence interval (95\% CI). Participants who consumed fruits for 6-7 days per week had lower risks of high triglycerides (OR: 0.66, 95\% CI: 0.58-0.75), dyslipidemia (OR: 0.77, 95\% CI: 0.68-0.86), and hyperlipidemia (OR: $0.72,95 \%$ CI: 0.63-0.81), compared to fruit consumption of 0-2 days per week. Risks of high triglycerides, dyslipidemia and hyperlipidemia of those who consumed fruits for $0.75-1.5$ servings each day also decreased, compared to the insufficient fruit intake. The combined effects of high frequency and moderate daily intake of fruit on lipid disorders did not change essentially. The associations were more evident in girls, younger children and those whose families had higher educational levels. Moderate fruit consumption was associated with lower odds of lipid disorders, predominantly in girls, younger participants, and those came from higher-educated families. These findings supported the health effect of moderate fruit intake frequently to improve the childhood lipid profiles.
\end{abstract}

Keywords: fruit consumption; lipid; dyslipidemia; children and adolescents; China

\section{Introduction}

Dyslipidemia, characterized by abnormal blood lipids and lipoprotein levels, is a recognized risk factor for cardiovascular disease [1]. Previous studies have observed a high prevalence of lipid disorders during both childhood and adolescence in China [2,3]. Observed from the 10-year trend of serum lipids, the pooled prevalence of dyslipidemia in Chinese children and adolescents was 28.9\% in 2014 [2], while in 2015, it increased to $31.6 \%$ in a nationally representative pediatric population with 129,426 participants [4]. It 
showed a younger-age trend during recent decades, and could be progressive into adulthood. Growing evidence indicated that, in children and adolescents, higher concentrations of low density lipoprotein-cholesterol (LDL-C), as well as lower concentrations of high density lipoprotein-cholesterol (HDL-C) were associated with higher risk of atherosclerosis in later life [5]. As such, screening children and adolescents for lipid profiles, which usually included the measurements of serum concentrations of total cholesterol (TC), triglycerides (TG), HDL-C and LDL-C, might have the potential to identify the affected subjects, reduce the burdens of long-term cholesterol through intervention, and postpone or prevent cardiovascular events during adulthood. The challenge was to maintain appropriate lipid levels at the right time, most commonly by early behavioral and lifestyle interventions for high-risk children and adolescents.

Previous work revealed that the nutritional and diet factors were important determinants for the development of childhood dyslipidemia [1,6], and therefore could be important targets for prevention strategies. Among the multiple diet factors, vegetables and fruits are important sources of a healthy diet. Notably, low fruit consumption is considered to be the fifth leading contributor to the global disease burden [7]. Growing observational evidence suggested that the fruit consumption might parallel the decrease in the risks of obesity, diabetes mellitus, and cardiovascular events in both childhood and adulthood [8,9]. Pan and his colleagues found a significant inverse association between healthy eating index score of fruits and the risk of metabolic syndrome (MS) among US adolescents, suggesting that a fruit-rich diet could exert beneficial effects in prevention of MS [10]. However, inadequate fruit consumption of children and adolescents worldwide despite the generally higher preference for consumption of fruits than vegetables [11], interventions to encourage fruit consumption during childhood and adolescence might therefore be an effective strategy in reducing disease burden.

So far, evidence for the effects of fruit consumption on lipid disorders had been limited to date. In 2013, a randomized controlled trial demonstrated no significant effect between increased fruits and vegetables consumption and healthier blood levels of TC [12]. In contrast, a cross-sectional study in Macao, China indicated that students who consumed less fruits suffered from a higher rate of low HDL-C level and elevated TG [13]. Besides, a fruits- and vegetables-rich diet was associated with a healthier metabolic profile, reflected by low concentrations of TC and LDL-C [14]. However, these studies only determined the consumption frequency of fruits, such as consuming fruits every day, not focused on the specific frequencies or average daily intake amount. Currently, the dietary guidelines for fruit intake were mostly based on adults, with the recommended daily fruit intake of $400 \mathrm{~g} \mathrm{[15],} \mathrm{while} \mathrm{there} \mathrm{was} \mathrm{no} \mathrm{consensus} \mathrm{regarding} \mathrm{daily} \mathrm{fruit} \mathrm{consumption} \mathrm{for} \mathrm{children}$ and adolescents [16], especially aimed at reducing adverse lipid profiles. Different from children and adolescents, adults could accumulate more complex environmental effects, while children might be more sugar-sensitive than adults. Guidelines based on findings in adults may therefore lead to ambiguity about fruit recommendations for pediatric cardiovascular and lipid health. In addition, fruit also contains a large amount of fructose, the accumulation of which is detrimental. Whether a causal link exists between natural sources of fructose present in fruits and the development of lipid disorders continues to be contested [17]. For these reasons, exploring the exact frequency and amount of fruit for children and adolescents to prevent lipid disorders was essential.

Given the role that fruit consumption played in pediatric health, we hereby investigated the relationship between fruit consumption and lipid profiles among children and adolescents aged 5-19 years, based on data of a large cross-sectional survey, which was conducted in seven provinces in China. The aim of the present study was to provide evidence on a satisfactory frequency and daily amount of fruit consumption to prevent deleterious lipid events for children and adolescents. 


\section{Materials and Methods}

\subsection{Study Population}

Data in this study came from the baseline of a multi-centered, cluster-controlled trial, aiming to prevent obesity in children and adolescents from seven provinces or cities of China (Hunan, Ningxia, Hunan, Chongqing, Liaoning, Shanghai, and Guangzhou; registration number: NCT02343588). The full trial protocol, including sampling procedure and measurements has been published previously in detail [18]. In brief, a multi-stage cluster random sampling method was used to determine the original study population. Firstly, several regions were randomly chosen from each province. Approximately 12-16 schools covering primary schools, junior high schools, and middle high schools were then randomly chosen from each region. In each school, two classes were randomly selected from each grade. All the students and their parents from selected classes were invited for participation. Among the original surveyed population of 16,637 participants aged 5-19 years whose physical examination and blood samples were available, 1862 participants were excluded from the present analysis because of missing information on fruit consumption, making the final sample size of 14,755 . The study has been approved by the ethical committee of the Peking University (number: IRB0000105213034). Written informed consent was obtained from both students and their parents or legal guardian in both waves.

\subsection{Questionnaire}

The children's questionnaire was performed in order to collect basic information and lifestyle behaviors. Besides, the parental self-administrated questionnaire included information about residence area, monthly household income, parental body mass index (BMI), and parental educational attainment. Both parental and children's questionnaires of children grade 1-3 were reported by parents. Children above the fourth grade would fill in children's questionnaire by themselves instructed by the class teacher.

Data regarding consumption of fruits and other eating behaviors were collected. As previously published [18-21], the frequency (days) and amount (serving per day) of dietary behaviors, including the total consumption of fruits, vegetables, meat and sugar-sweetened beverages (SSBs) over the past 7 days, were investigated. Participants were asked “How many days, over the past 7 days, have you eaten fruit/vegetables/meat or drunk SSB? How many servings in one day?" [18-21]. Previous studies determined the consumption frequency of fruits as consuming fruits every day [13], in order to avoid extreme few samples in each group, we categorized the frequency of those dietary behaviors into three groups of "0-2 days/week", "3-5 days/week", and "6-7 days/week". To better understand the intake of fruit/vegetable, one serving was defined as the size of an ordinary adult's closed fist (Supplementary Figure S1) and roughly equaled a medium-sized apple or orange $(\approx 200 \mathrm{~g})$ [22], which has been described in detail elsewhere [21]. As set in the questionnaire, SSB included Coca-Cola, Sprite, orange juice, Nutrition Express, and Red Bull [20]. One serving of SSB was determined as a canned beverage (approximately $250 \mathrm{~mL}$ ), while one portion of meat equaled the size of an adult's palm (approximately $100 \mathrm{~g}$ ) [23]. The daily dietary intake was calculated as: average daily intake $=$ (days of consumption $\times$ servings in those days $) / 7$.

According to the Dietary Guidelines for Chinese School-age Children 2016 [24], inadequate daily fruit intake was defined as eating fruits less than $150 \mathrm{~g}$ each day, and the recommended daily intake was approximately 250-350 g for the pediatric population. The amount of daily fruit intake was therefore categorized into groups of " $<0.75$ servings/day (approximately <150 g)”, “0.75-1.5 servings/day ( $\geq 0.75$ and $<1.5$ servings/day) (approximately 150-300 g)", and “ $\geq 1.5$ servings/day (approximately $\geq 300 \mathrm{~g}$ )".

Information about the child's physical activity was collected with the International Physical Activity Questionnaire-Short Form (IPAQ-SF) [25], which is more suitable for population surveillance and large-scale studies [26]. Instructed by trained project members, all recruited participants were asked to report their frequency (days) and duration (hours and minutes) of moderate to vigorous-intensity physical activities 
(MVPA) over the past 7 days, and the average time for MVPA per day was calculated as: average daily time $=($ days of physical activity $\times$ duration in those days $) / 7$.

Parents were asked to report their height $(\mathrm{cm})$ and weight $(\mathrm{kg})$, while body mass index (BMI) was calculated as the weight $(\mathrm{kg})$ divided by the square of the height $\left(\mathrm{m}^{2}\right)$. According to the criteria established by the Working Group on Obesity in China (WGOC) for Chinese adults [27], BMI cut-offs of 24 and $28 \mathrm{~kg} / \mathrm{m}^{2}$ were applied to categorize each parent into the categories of normal, overweight and obesity. Parental educational attainment was surveyed and grouped into "primary school or below", "secondary or equivalent" and "junior college or above". In addition, the residence area was divided into "rural" and "urban", and monthly household income was defined with the sum of monthly income (in $\mathrm{CNY}$ ) of all household members and then classified into $<5000$, or $\geq 5000 \mathrm{CNY}$.

\subsection{Anthropometric Measurements}

Anthropometric measurements were conducted by trained project members according to standardized procedure, and the measuring instruments were similar at all study sites. Children were required to stand straight in light clothing and without shoes for the measurements. Height was measured using the portable stadiometer with $0.1 \mathrm{~cm}$ precision, weight was measured to the nearest $0.1 \mathrm{~kg}$ by a Lever type weight scale. Every indicator was measured twice, and the average level of the two measurements was calculated for final analyses. BMI was calculated as body weight $(\mathrm{kg})$ divided by height $(\mathrm{m})$ squared.

\subsection{Blood Sample Collection and Detection}

Venous blood sample was obtained in the morning after an overnight (at least $8 \mathrm{~h}$ ) fasting. Children were asked to rest for at least $10 \mathrm{~min}$ before blood sample collection. Blood specimens were then transported in a chilled insulated container immediately, and then centrifuged at $3000 \mathrm{rpm}$ for $10 \mathrm{~min}$ and then frozen at $-80^{\circ} \mathrm{C}$. All plasma samples were transported by air in dry ice to the laboratory in Beijing, where the samples were stored at $-80^{\circ} \mathrm{C}$ before laboratory detections. All the biochemical analyses were conducted at a biomedical analyses company accredited by Peking University [18]. Lipid profiles were measured with an autoanalyzer (TBA-120FR, Toshiba, Tokyo, Japan), with TC and TG assayed by enzymatic method, while LDL-C and HDL-C measured by clearance method.

\subsection{Definition of Abnormal Lipid Profile and Overweight/Obesity}

Following the 2011 Expert Panel on Integrated Guidelines for Cardiovascular Health and Risk Reduction in Children and Adolescents [28], high TG was defined as TG $\geq 1.13 \mathrm{mmol} / \mathrm{L}$ for children aged 9 years or younger, and TG $\geq 1.47 \mathrm{mmol} / \mathrm{L}$ for adolescents aged 10 years or older. High TC was considered as TC $\geq 5.18 \mathrm{mmol} / \mathrm{L}$, high LDL-C was regarded as LDL-C $\geq 3.37 \mathrm{mmol} / \mathrm{L}$, and low HDL-C referred to HDL-C $\leq 1.04 \mathrm{mmol} / \mathrm{L}$. Because non-HDL-C levels showed better prediction of persistent dyslipidemia, the prevalence of high non-HDL-C levels was also presented, as was calculated as the difference between TC level and the HDL-C level. High non-HDL-C were determined as non-HDL-C $\geq 3.76 \mathrm{mmol} / \mathrm{L}$ [28]. A participant with one or more abnormal lipid levels (high LDC-C, low HDL-C, high TG, or high TC) was defined as having dyslipidemia. In addition, hyperlipidemia was defined with the presence of high TG or high TC levels [28].

In accordance with the guideline of the Working Group on Obesity in China, participants with age- and sex-specific BMI $<85$ th percentile were considered as non-overweight/ obese, and those with $\geq 85$ th percentile BMI were considered as overweight/obese [29].

\subsection{Statistical Analysis}

Data were expressed as mean \pm SD for continuous variables and number $(\%)$ for categorical variables. We used Bonferroni multiple comparison methods to examine the differences in prevalence of adverse lipid profiles between groups. The significance of Bonferroni formula was set at $\alpha=0.017$. To understand whether some demographics, dietary or lifestyle factors could modify the associations, two separate logistic regression 
models were applied to estimate the odds ratio (OR) and 95\% confidence interval (95\% CI) between fruit consumption and lipid profile. In model 1, age and residence area were adjusted. In model 2, several additional confounding factors, including sex (boy, girl), BMI values, ethnicity (Han, Hui, Tibetan, Mongolian and Other), monthly household income $(<5000$, or $\geq 5000 \mathrm{CNY}$ ), parental weight status (normal, overweight or obesity), parental educational attainment (primary school or below, secondary or equivalent, junior college or above), vegetable consumption, sugar-sweetened beverages consumption, meat consumption and physical activity were included. Additionally, previous studies had shown that lipid levels were dependent on age and sexual maturation [30], and could be influenced by the difference of socioeconomic support mainly determined by the degree of their parents' education [31]. Besides, BMI values were closely related with lipid health. Therefore, stratified analyses were conducted according to sex, age, parental educational attainment and BMI values.

All analyses were performed using SAS software (version 9.4; SAS Institute, lnc., Cary, NC, USA). A two-sided $p$ value $<0.05$ was considered statistically significant.

\section{Results}

\subsection{General Characteristics}

A total of 7420 boys and 7335 girls were included in the final analysis. Table 1 showed the characteristics of the study population. The mean age was $11.15 \pm 3.29$ years old and their average BMI value was $18.74 \pm 3.85 \mathrm{~kg} / \mathrm{m}^{2}$. The majority of the study population was Han ethnicity (92.56\%), and most of their parents belonged to the normal weight status (paternal: $56.88 \%$; maternal: $79.49 \%$ ). In addition, $35.33 \%$ of the children's fathers received junior college or above education, $58.33 \%$ for secondary or equivalent and $6.34 \%$ for primary school or below. A similar trend was observed for maternal educational attainment. It was worth noting that most of them had a monthly household income of less than 5000 yuan. Besides, the average concentrations of TC, TG, LDL-C, HDL-C and non-HDL-C were $3.89 \pm 0.88$, $1.09 \pm 0.77,2.01 \pm 0.69,1.90 \pm 1.35$ and $1.99 \pm 1.56 \mathrm{mmol} / \mathrm{L}$, respectively.

Table 1. Baseline characteristic of included population.

\begin{tabular}{|c|c|c|c|c|}
\hline Characteristics & Total Population $(n=14,755)$ & Boys $(n=7420)$ & Girls ( $n=7335)$ & $p$-Value \\
\hline Age, year & $11.15 \pm 3.29$ & $11.13 \pm 3.25$ & $11.16 \pm 3.33$ & 0.533 \\
\hline Weight, kg & $42.27 \pm 15.91$ & $44.09 \pm 17.46$ & $40.45 \pm 13.94$ & $<0.01$ \\
\hline Height, m & $1.48 \pm 0.17$ & $1.49 \pm 0.18$ & $1.46 \pm 0.15$ & $<0.01$ \\
\hline BMI, $\mathrm{kg} / \mathrm{m}^{2}$ & $18.74 \pm 3.85$ & $19.04 \pm 4.04$ & $18.45 \pm 3.63$ & $<0.01$ \\
\hline Residence area $(n, \%)$ & & & & 0.701 \\
\hline Urban & $7860(53.27 \%)$ & $3941(53.11 \%)$ & $3919(53.43 \%)$ & \\
\hline Rural & $6895(46.73 \%)$ & $3479(46.89 \%)$ & $3416(46.57 \%)$ & \\
\hline Ethnicity $(n, \%)$ & & & & 0.066 \\
\hline Han & $13,657(92.56 \%)$ & $6876(92.67 \%)$ & $6781(92.45 \%)$ & \\
\hline Hui & $528(3.58 \%)$ & $248(3.34 \%)$ & $280(3.82 \%)$ & \\
\hline Tibetan & $33(0.22 \%)$ & $14(0.19 \%)$ & $19(0.26 \%)$ & \\
\hline Mongolian & $187(1.27 \%)$ & $110(1.48 \%)$ & $77(1.05 \%)$ & \\
\hline Other & $350(2.37 \%)$ & $172(2.32 \%)$ & $178(2.43 \%)$ & \\
\hline Paternal weight status, $n(\%)$ & & & & 0.451 \\
\hline Normal & $8393(56.88 \%)$ & $4252(57.30 \%)$ & $4141(56.46 \%)$ & \\
\hline Overweight & $4834(32.76 \%)$ & $2395(32.28 \%)$ & $2439(33.25 \%)$ & \\
\hline Obesity & $1528(10.36 \%)$ & $773(10.42 \%)$ & $755(10.29 \%)$ & \\
\hline Maternal weight status, $n(\%)$ & & & & 0.002 \\
\hline Normal & $11,729(79.49 \%)$ & $5983(80.63 \%)$ & $5746(78.34 \%)$ & \\
\hline Overweight & $2462(16.69 \%)$ & $1176(15.85 \%)$ & $1286(17.53 \%)$ & \\
\hline Obesity & $564(3.82 \%)$ & $261(3.52 \%)$ & $303(4.13 \%)$ & \\
\hline Paternal educational attainment, $n(\%)$ & & & & 0.229 \\
\hline Primary school or below & $936(6.34 \%)$ & $451(6.08 \%)$ & $485(6.61 \%)$ & \\
\hline Secondary or equivalent & $8606(58.33 \%)$ & $4371(58.91 \%)$ & $4235(57.74 \%)$ & \\
\hline
\end{tabular}


Table 1. Cont.

\begin{tabular}{|c|c|c|c|c|}
\hline Characteristics & Total Population $(n=14,755)$ & Boys $(n=7420)$ & Girls $(n=7335)$ & $p$-Value \\
\hline Junior college or above & $5213(35.33 \%)$ & $2598(35.01 \%)$ & $2615(35.65 \%)$ & \\
\hline Maternal educational attainment, $n(\%)$ & & & & 0.385 \\
\hline Primary school or below & $1277(8.65 \%)$ & $619(8.34 \%)$ & $658(8.97 \%)$ & \\
\hline Secondary or equivalent & $8429(57.13 \%)$ & $4246(57.22 \%)$ & $4183(57.03 \%)$ & \\
\hline Junior college or above & $5049(34.22 \%)$ & $2555(34.43 \%)$ & $2494(34.00 \%)$ & \\
\hline Monthly household income, $n(\%)$ & & & & 0.220 \\
\hline$<5000$ yuan & $12,402(84.05 \%)$ & $6264(84.42 \%)$ & $6138(83.68 \%)$ & \\
\hline$\geq 5000$ yuan & $2353(15.95 \%)$ & $1156(15.58 \%)$ & $1197(16.32 \%)$ & \\
\hline \multicolumn{5}{|l|}{$\begin{array}{l}\text { Frequency of consumption } \\
\text { (days per week) }\end{array}$} \\
\hline Fruit & $4.93 \pm 2.11$ & $4.75 \pm 2.19$ & $5.11 \pm 2.02$ & $<0.01$ \\
\hline Vegetables & $6.02 \pm 1.82$ & $5.99 \pm 1.85$ & $6.06 \pm 1.79$ & 0.011 \\
\hline Sugar-sweetened beverages & $1.63 \pm 1.78$ & $1.84 \pm 1.91$ & $1.41 \pm 1.62$ & $<0.01$ \\
\hline Meat & $5.01 \pm 2.24$ & $5.21 \pm 2.18$ & $4.80 \pm 2.28$ & $<0.01$ \\
\hline \multicolumn{5}{|l|}{$\begin{array}{l}\text { Average daily consumption } \\
\text { (servings per day) }\end{array}$} \\
\hline Fruit & $1.33 \pm 1.14$ & $1.32 \pm 1.19$ & $1.33 \pm 1.08$ & 0.567 \\
\hline Vegetables & $1.87 \pm 1.50$ & $1.89 \pm 1.53$ & $1.85 \pm 1.46$ & 0.148 \\
\hline Sugar-sweetened beverages & $0.46 \pm 0.84$ & $0.56 \pm 0.96$ & $0.36 \pm 0.68$ & $<0.01$ \\
\hline Meat & $1.24 \pm 1.30$ & $1.39 \pm 1.42$ & $1.09 \pm 1.15$ & $<0.01$ \\
\hline $\begin{array}{c}\text { Frequency of physical activity, } \\
\text { days/week }\end{array}$ & $3.35 \pm 2.53$ & $3.47 \pm 2.55$ & $3.23 \pm 2.50$ & $<0.01$ \\
\hline $\begin{array}{c}\text { Average daily physical activity, hours } \\
\text { and minutes }\end{array}$ & $0.37 \pm 0.82$ & $0.42 \pm 0.89$ & $0.32 \pm 0.74$ & $<0.01$ \\
\hline $\mathrm{TC}, \mathrm{mmol} / \mathrm{L}$ & $3.89 \pm 0.88$ & $3.83 \pm 0.87$ & $3.94 \pm 0.89$ & $<0.01$ \\
\hline $\mathrm{TG}, \mathrm{mmol} / \mathrm{L}$ & $1.09 \pm 0.77$ & $1.04 \pm 0.74$ & $1.14 \pm 0.79$ & $<0.01$ \\
\hline LDL-C, mmol/L & $2.01 \pm 0.69$ & $1.98 \pm 0.67$ & $2.03 \pm 0.70$ & $<0.01$ \\
\hline HDL-C, mmol/L & $1.90 \pm 1.35$ & $1.90 \pm 1.38$ & $1.90 \pm 1.33$ & 0.993 \\
\hline Non-HDL-C, mmol/L & $1.99 \pm 1.56$ & $1.94 \pm 1.58$ & $2.04 \pm 1.54$ & $<0.01$ \\
\hline
\end{tabular}

Abbreviation: BMI, body mass index; TC, total cholesterol; TG, triglycerides; LDL-C, low density lipoproteincholesterol; HDL-C, high density lipoprotein-cholesterol.

Furthermore, girls tended to consume fruits and vegetables more frequently per week $(p<0.05)$, while boys were more likely to consume sugar-sweetened beverages and meat $(p<0.01)$. As for the average daily consumption, intakes of fruits $(p=0.567)$ and vegetables $(p=0.148)$ were not significantly different among sex-specific groups, but boys consumed more sugar-sweetened beverages and meat daily (both $p<0.01$ ). Of note, boys were prone to exercise more frequently and for longer duration (both $p<0.01$ ).

\subsection{The Prevalence of Abnormal Lipid Profile}

The prevalence of abnormal lipid profile was present in Supplementary Table S1. Compared with participants consuming fruits for only 0-2 days/week, the prevalence of high TG, low HDL-C, dyslipidemia, and hyperlipidemia tended to be low among those who consumed fruits more frequently. Notably, similar lower prevalence for these lipid disorders was observed in the group of average daily fruit intake of $0.75-1.5$ servings, compared to those with daily fruit consumption of $\geq 1.5$ servings. Similar trends of prevalence of adverse lipid profiles were found for boys and girls, respectively.

\subsection{Association between Fruit Consumption and Lipid Profile}

The associations between fruit consumption and the odds of abnormal lipid profile were present in Table 2, high frequency of fruit intake per week was associated with lower possibility of unfavorable lipid profiles. In the basically adjusted model 1, the ORs $(95 \% \mathrm{CI})$ of frequent fruit consumption (6-7 days/week) were $0.74(0.66-0.83)$, $0.83(0.75-0.92)$ and $0.79(0.70-0.88)$ for the risk of high TG, dyslipidemia and hyperlipi- 
demia, respectively. After controlling for potential covariates included in model 2, the results did not change essentially.

Table 2. Multivariate odds ratios (OR) and 95\% confidence intervals (CI) of fruit consumption and lipid profile $(n=14,755)$.

\begin{tabular}{|c|c|c|c|c|c|c|}
\hline \multirow{2}{*}{ Lipid Profile } & \multicolumn{3}{|c|}{ Frequency of Fruit Consumption } & \multicolumn{3}{|c|}{ Average Daily Fruit Consumption } \\
\hline & 0-2 Days/Week & 3-5 Days/Week & 6-7 Days/Week & $<0.75$ Servings/Day & 0.75-1.5 Servings/Day & $\geq 1.5$ Servings/Day \\
\hline \multicolumn{7}{|c|}{ Model $1^{1}$} \\
\hline High TC & 1 (Reference) & $1.09(0.86-1.38)$ & $1.17(0.93-1.46)$ & 1 (Reference) & $1.01(0.85-1.20)$ & $0.93(0.78-1.12)$ \\
\hline High TG & 1 (Reference) & $0.88(0.79-0.99) *$ & $0.74(0.66-0.83) * *$ & 1 (Reference) & $0.92(0.83-1.01)$ & $1.09(0.99-1.21)$ \\
\hline High LDL-C & 1 (Reference) & $1.14(0.84-1.55)$ & $1.13(0.84-1.53)$ & 1 (Reference) & $0.93(0.75-1.17)$ & $0.86(0.67-1.09)$ \\
\hline Low HDL-C & 1 (Reference) & $0.96(0.84-1.11)$ & $0.88(0.76-1.01)$ & 1 (Reference) & $0.94(0.83-1.05)$ & $1.06(0.94-1.19)$ \\
\hline $\begin{array}{c}\text { High } \\
\text { non-HDL-C }\end{array}$ & 1 (Reference) & $0.99(0.78-1.26)$ & $1.05(0.83-1.32)$ & 1 (Reference) & $1.07(0.89-1.28)$ & $0.90(0.74-1.10)$ \\
\hline Dyslipidemia & 1 (Reference) & $0.97(0.87-1.07)$ & $0.83(0.75-0.92) * *$ & 1 (Reference) & $0.93(0.86-1.01)$ & $1.05(0.96-1.15)$ \\
\hline Hyperlipidemia & 1 (Reference) & $0.92(0.82-1.03)$ & $0.79(0.70-0.88)^{* *}$ & 1 (Reference) & $0.92(0.84-1.01)$ & $1.07(0.97-1.17)$ \\
\hline \multicolumn{7}{|c|}{ Model $2^{2}$} \\
\hline High TC & 1 (Reference) & $1.13(0.88-1.45)$ & $1.18(0.92-1.50)$ & 1 (Reference) & $1.01(0.85-1.22)$ & $0.94(0.77-1.14)$ \\
\hline High TG & 1 (Reference) & $0.84(0.74-0.96)$ * & $0.66(0.58-0.75)^{* *}$ & 1 (Reference) & $0.87(0.78-0.97)^{* *}$ & $1.00(0.90-1.13)$ \\
\hline High LDL-C & 1 (Reference) & $1.23(0.89-1.71)$ & $1.09(0.79-1.51)$ & 1 (Reference) & $0.92(0.73-1.17)$ & $0.80(0.62-1.03)$ \\
\hline Low HDL-C & 1 (Reference) & $1.02(0.88-1.19)$ & $0.88(0.76-1.03)$ & 1 (Reference) & $0.91(0.80-1.03)$ & $1.00(0.87-1.14)$ \\
\hline $\begin{array}{c}\text { High } \\
\text { non-HDL-C }\end{array}$ & 1 (Reference) & $1.07(0.83-1.38)$ & $1.05(0.82-1.35)$ & 1 (Reference) & $1.08(0.89-1.31)$ & $0.87(0.70-1.07)$ \\
\hline Dyslipidemia & 1 (Reference) & $0.95(0.85-1.06)$ & $0.77(0.68-0.86)^{* *}$ & 1 (Reference) & $0.88(0.81-0.97)^{* *}$ & $0.98(0.89-1.08)$ \\
\hline Hyperlipidemia & 1 (Reference) & $0.88(0.78-1.00)$ & $0.72(0.63-0.81)^{* *}$ & 1 (Reference) & $0.88(0.80-0.97) * *$ & $1.00(0.90-1.11)$ \\
\hline
\end{tabular}

${ }^{1}$ Model 1: adjusted for age and residence area. ${ }^{2}$ Model 2: additionally adjusted for sex, BMI values, ethnicity, incomes, parental educational attainment, parental weight, vegetable consumption, sugar-sweetened beverages consumption, meat consumption and physical activity. TC, total cholesterol; TG, triglycerides; LDL-C, low density lipoprotein-cholesterol; HDL-C, high density lipoprotein-cholesterol. ${ }^{*} p<0.05,{ }^{* *} p<0.01$.

In addition, adjusting for factors in model 2, participants who consumed fruits moderately for $0.75-1.5$ servings each day were estimated to have 0.87 times (OR: 0.87 , 95\% CI: 0.78-0.97), 0.88 times (OR: $0.88,95 \%$ CI: $0.81-0.97$ ) and 0.88 times (OR: 0.88 , 95\% CI: 0.80-0.97) lower odds of high TG, dyslipidemia and hyperlipidemia compared with their counterparts with insufficient fruit consumption each time. There were no statistically significant associations between fruit consumption over 1.5 servings/day and risks of high TC, high LDL-C, low HDL-C and high non-HDL-C.

Apart from this, consuming fruits for 6-7 days/week combined with moderate intake of $0.75-1.5$ servings each day could improve adverse lipid profiles (Table 3 ). Children and adolescents who consumed fruits moderately of $0.75-1.5$ servings/day for 6-7 days/week had lower risks of high TG, low HDL-C, dyslipidemia and hyperlipidemia (all $p<0.05$ ).

Table 3. Combined effects of frequencies and daily intake of fruit consumption on adverse lipid profile $(n=14,755)$.

\begin{tabular}{ccccc}
\hline & & \multicolumn{3}{c}{ 6-7 Days/Week $(\boldsymbol{n}=\mathbf{6 8 2 9})$} \\
\cline { 3 - 5 } Lipid Profile & Unselected Population & $\begin{array}{c}<\mathbf{0 . 7 5} \text { Servings/Day } \\
(\boldsymbol{n = 6 1 )}\end{array}$ & $\begin{array}{c}\mathbf{0 . 7 5 - 1 . 5} \text { Servings/Day } \\
(\boldsymbol{n}=\mathbf{3 0 3 6 )}\end{array}$ & $\begin{array}{c}\mathbf{1 . 5} \text { Servings/Day } \\
(\boldsymbol{n}=\mathbf{3 7 3 2})\end{array}$ \\
\hline High TC & 1 (Reference) & $0.48(0.11-2.04)$ & $1.10(0.92-1.32)$ & $1.01(0.85-1.21)$ \\
High TG & 1 (Reference) & $0.78(0.36-1.68)$ & $0.68(0.61-0.77)$ & $0.94(0.85-1.05)$ \\
High LDL-C & 1 (Reference) & $0.71(0.15-3.23)$ & $1.03(0.81-1.31)$ & $0.90(0.71-1.13)$ \\
Low HDL-C & 1 (Reference) & $0.86(0.35-2.09)$ & $0.83(0.73-0.96)^{*}$ & $0.97(0.86-1.09)$ \\
High non-HDL-C & 1 (Reference) & $0.75(0.21-2.73)$ & $1.12(0.92-1.36)$ & $0.91(0.75-1.09)$ \\
Dyslipidemia & 1 (Reference) & $0.69(0.36-1.33)$ & $0.76(0.69-0.83)^{* *}$ & $0.95(0.87-1.04)$ \\
Hyperlipidemia & 1 (Reference) & $0.68(0.33-1.41)$ & $0.72(0.65-0.81)^{* *}$ & $0.96(0.88-1.06)$ \\
\hline
\end{tabular}

Adjusted in Model 2: including age, residence area, sex, BMI values, ethnicity, incomes, parental educational attainment, parental weight, vegetable consumption, sugar-sweetened beverages consumption, meat consumption and physical activity. TC, total cholesterol; TG, triglycerides; LDL-C, low density lipoprotein-cholesterol; HDL-C, high density lipoprotein-cholesterol. ${ }^{*} p<0.05,{ }^{* *} p<0.01$. 


\subsection{Stratified Analyses}

The results of stratified analyses were similar with the main results (Figures 1,2 and S1). The more frequent of fruit intake was associated with lower odds of high TG, dyslipidemia and hyperlipidemia both in boys and girls, while moderately consuming fruits each day could only lower the likelihood of lipid disorders among girls (Figure 1). Notably, compared to the reference group, significantly lower likelihoods of high TG and hyperlipidemia were observed among all participants aged 5-19 years old who consumed fruits more frequently, but only 5-14-year-old children and adolescents had a lower risk of dyslipidemia $(p<0.01)$. However, when considering consuming fruits moderately of 0.75-1.5 servings per day, only 5-to-9-year-old children were estimated to have 0.80 times (OR: 0.80, 95\% CI: 0.66-0.96), 0.79 times (OR: 0.79 , 95\% CI: 0.68-0.92) and 0.77 times (OR: 0.77, 95\% CI: 0.66-0.91) lower odds of high TG, dyslipidemia and hyperlipidemia, respectively (Figure 2).

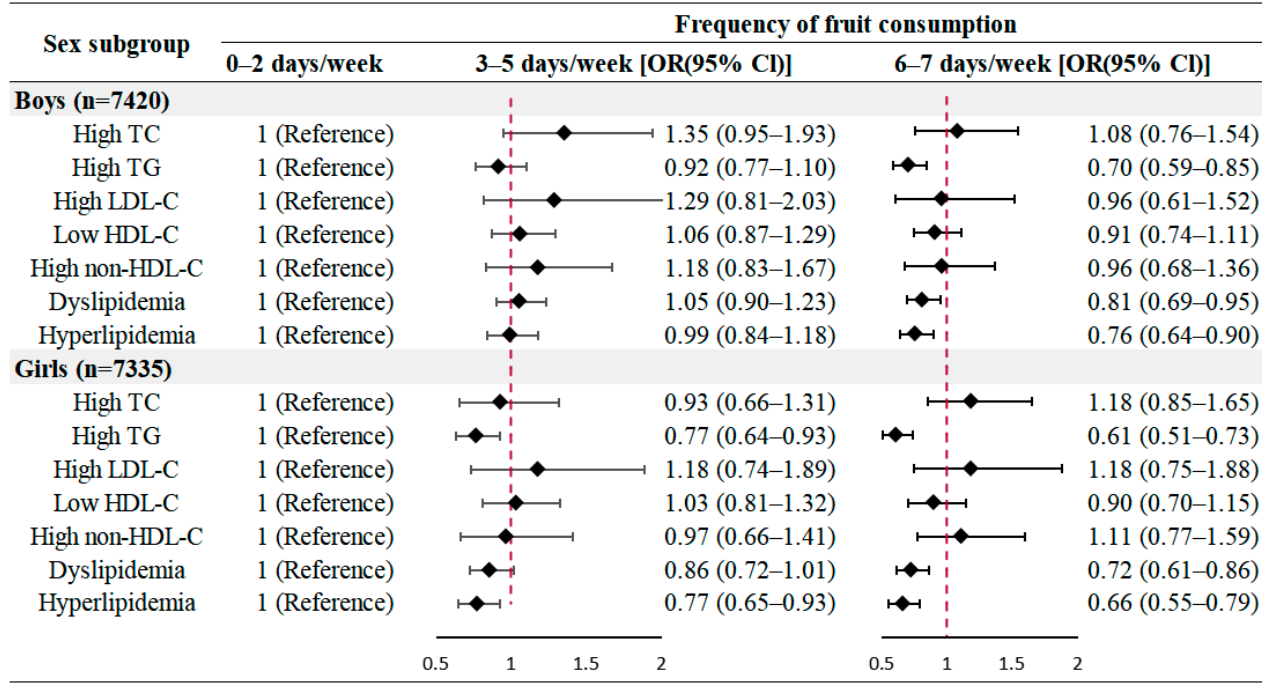

(a)

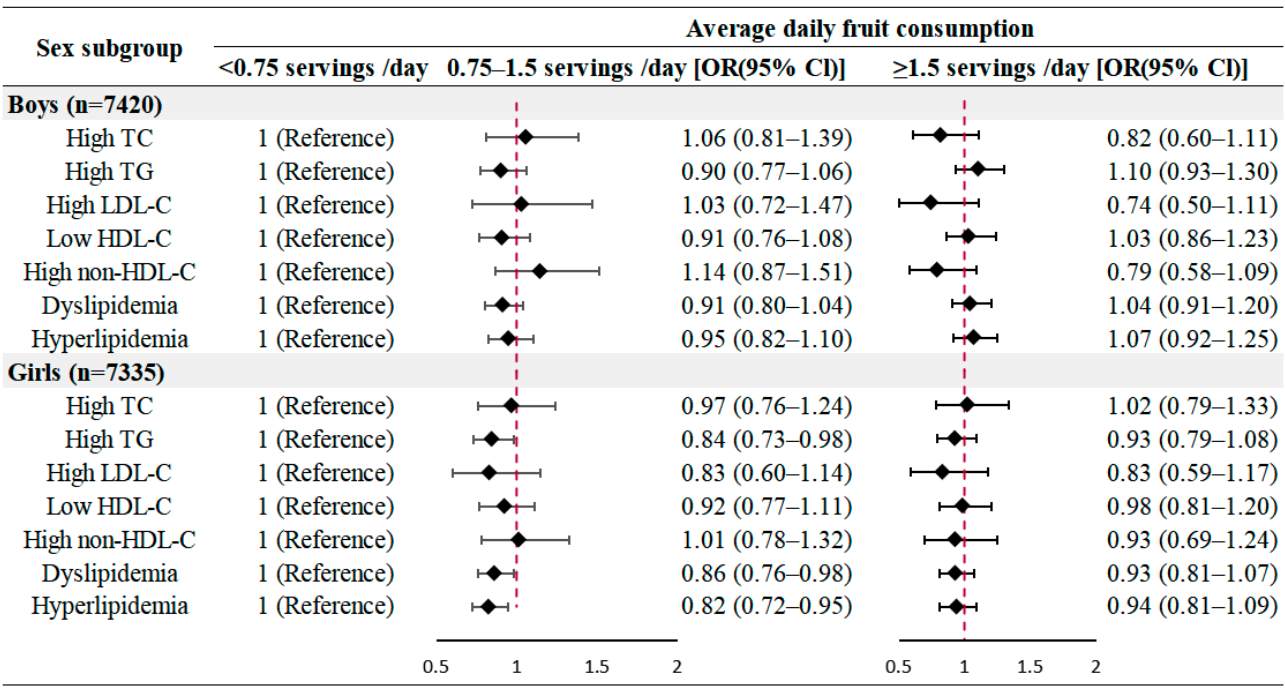

(b)

Figure 1. Sex-specific analysis of the associations between fruit consumption and lipid profile (adjusted for age, BMI values, residence area, ethnicity, incomes, parental educational attainment, parental weight, vegetable consumption, sugar-sweetened beverages consumption, meat consumption and physical activity) (a) Frequency of fruit consumption; (b) Average daily fruit consumption. TC, total cholesterol; TG, triglycerides; LDL-C, low density lipoprotein-cholesterol; HDL-C, high density lipoprotein-cholesterol; diamond symbol, point estimates; red dashed line, invalid line. 


\begin{tabular}{|c|c|c|c|c|c|}
\hline \multirow{2}{*}{ Age subgroup } & \multirow[b]{2}{*}{ 0-2 days/week } & \multicolumn{4}{|c|}{ Frequency of fruit consumption } \\
\hline & & \multicolumn{2}{|c|}{ 3-5 days/week $[\mathrm{OR}(95 \% \mathrm{Cl})]$} & \multicolumn{2}{|c|}{ 6-7 days/week [OR(95\% CI)] } \\
\hline \multicolumn{6}{|c|}{ 5-9 years old $(n=5646)$} \\
\hline High TC & 1 (Reference) & $\mapsto$ & $0.90(0.62-1.29)$ & $\mapsto \frac{1}{1}$ & $0.85(0.60-1.22)$ \\
\hline High TG & 1 (Reference) & $\rightarrow$ & $0.87(0.69-1.11)$ & & $0.69(0.54-0.87)$ \\
\hline High LDL-C & 1 (Reference) & $\mapsto$ & $1.19(0.72-1.96)$ & • & $0.90(0.55-1.48)$ \\
\hline Low HDL-C & 1 (Reference) & 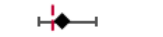 & $1.10(0.80-1.53)$ & & $1.04(0.75-1.43)$ \\
\hline High non-HDL-C & 1 (Reference) & $\mapsto$ & $1.07(0.71-1.62)$ & セロー & $0.91(0.60-1.36)$ \\
\hline Dyslipidemia & 1 (Reference) & $1-1$ & $0.92(0.75-1.13)$ & iأ-i & $0.74(0.61-0.90)$ \\
\hline Hyperlipidemia & 1 (Reference) & 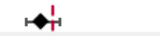 & $0.87(0.70-1.08)$ & $\leftrightarrow$ ! & $0.69(0.56-0.86)$ \\
\hline \multicolumn{6}{|c|}{10 -14 years old $(n=5776)$} \\
\hline High TC & 1 (Reference) & $\stackrel{1}{a} \bullet$ & $1.33(0.86-2.06)$ & & $1.49(0.97-2.29)$ \\
\hline High TG & 1 (Reference) & -1 & $0.84(0.69-1.02)$ & $\infty$ & $0.65(0.53-0.79)$ \\
\hline High LDL-C & 1 (Reference) & $\longmapsto$ & $1.15(0.68-1.96)$ & $\mapsto$ & $1.16(0.69-1.95)$ \\
\hline Low HDL-C & 1 (Reference) & $1+1$ & $0.97(0.77-1.23)$ & 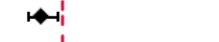 & $0.75(0.60-0.95)$ \\
\hline High non-HDL-C & 1 (Reference) & $\longmapsto$ & $1.05(0.69-1.60)$ & & $1.16(0.78-1.75)$ \\
\hline Dyslipidemia & 1 (Reference) & 14 & $0.94(0.78-1.12)$ & $\omega$ & $0.74(0.61-0.88)$ \\
\hline Hyperlipidemia & 1 (Reference) & 14 & $0.89(0.73-1.07)$ & Wi & $0.73(0.60-0.88)$ \\
\hline \multicolumn{6}{|c|}{$15-19$ years old $(n=3333)$} \\
\hline High TC & 1 (Reference) & & $1.42(0.82-2.46)$ & & $1.66(0.97-2.85)$ \\
\hline High TG & 1 (Reference) & -4 & $0.79(0.61-1.03)$ & $\rightarrow 1$ & $0.60(0.47-0.78)$ \\
\hline High LDL-C & 1 (Reference) & & $-1.55(0.72-3.34)$ & & $1.63(0.77-3.46)$ \\
\hline Low HDL-C & 1 (Reference) & $n_{\infty}$ & $1.09(0.83-1.43)$ & & $1.02(0.77-1.34)$ \\
\hline High non-HDL-C & 1 (Reference) & $\longmapsto$ & $1.17(0.70-1.96)$ & & $1.34(0.81-2.20)$ \\
\hline Dyslipidemia & 1 (Reference) & $\mapsto-1$ & $1.02(0.82-1.27)$ & is & $0.86(0.69-1.07)$ \\
\hline \multirow[t]{2}{*}{ Hyperlipidemia } & 1 (Reference) & 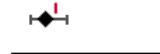 & $0.89(0.69-1.14)$ & $-\infty$ & $0.70(0.54-0.90)$ \\
\hline & & $1 \quad 1.5$ & $2.5 \quad 3$ & $\begin{array}{llll}0.5 & 1 & 1.5 & 2\end{array}$ & 3 \\
\hline
\end{tabular}

(a)

\begin{tabular}{|c|c|c|c|c|c|}
\hline \multirow{2}{*}{ Age subgroup } & \multirow[b]{2}{*}{$<0.75$ servings /day } & \multicolumn{4}{|c|}{ Average daily fruit consumption } \\
\hline & & $0.75-1.5$ serving & ay $[\mathrm{OR}(95 \% \mathrm{Cl})]$ & $\geq 1.5$ servings & OR(95\% Cl)] \\
\hline \multicolumn{6}{|c|}{ 5-9 years old $(n=5646)$} \\
\hline High TC & 1 (Reference) & $\mapsto \dot{1}$ & $0.73(0.56-0.96)$ & $\mapsto \begin{array}{c}1 \\
\end{array}$ & $0.84(0.63-1.13)$ \\
\hline High TG & 1 (Reference) & $\mapsto \mapsto \mid$ & $0.80(0.66-0.96)$ & 田 & $0.93(0.76-1.13)$ \\
\hline High LDL-C & 1 (Reference) & $\mapsto \quad$ & $0.70(0.50-1.00)$ & $\mapsto \longrightarrow$ & $0.72(0.49-1.06)$ \\
\hline Low HDL-C & 1 (Reference) & $\mapsto$ & $1.05(0.82-1.33)$ & 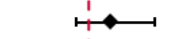 & $1.15(0.88-1.50)$ \\
\hline High non-HDL-C & 1 (Reference) & $\mapsto 1$ & $0.85(0.63-1.15)$ & $\mapsto 1$ & $0.88(0.63-1.23)$ \\
\hline Dyslipidemia & 1 (Reference) & $\mapsto \mapsto \mid$ & $0.79(0.68-0.92)$ & मִ' & $0.91(0.77-1.07)$ \\
\hline Hyperlipidemia & 1 (Reference) & 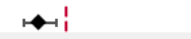 & $0.77(0.66-0.91)$ & (1) & $0.87(0.73-1.04)$ \\
\hline \multicolumn{6}{|c|}{$10-14$ years old $(n=5776)$} \\
\hline High TC & 1 (Reference) & $\longrightarrow$ & $1.33(0.96-1.83)$ & $\longmapsto$ & $1.05(0.74-1.47)$ \\
\hline High TG & 1 (Reference) & $\mapsto$ & $0.90(0.76-1.07)$ & & $0.98(0.82-1.16)$ \\
\hline High LDL-C & 1 (Reference) & & $1.23(0.82-1.84)$ & & $0.89(0.57-1.37)$ \\
\hline Low HDL-C & 1 (Reference) & $\mapsto$ & $0.80(0.66-0.98)$ & $\mapsto$ & $0.93(0.76-1.14)$ \\
\hline High non-HDL-C & 1 (Reference) & & $1.51(1.09-2.11)$ & & $1.01(0.71-1.45)$ \\
\hline Dyslipidemia & 1 (Reference) & $r$ & $0.92(0.80-1.07)$ & & $0.99(0.85-1.15)$ \\
\hline Hyperlipidemia & 1 (Reference) & $-\frac{1}{1}$ & $0.94(0.80-1.10)$ & & $1.03(0.87-1.21)$ \\
\hline \multicolumn{6}{|c|}{$15-19$ years old $(n=3333)$} \\
\hline High TC & 1 (Reference) & & $1.47(0.99-2.17)$ & & $1.15(0.72-1.84)$ \\
\hline High TG & 1 (Reference) & $\mapsto \bullet$ & $0.82(0.65-1.04)$ & $\mapsto$ & $0.93(0.71-1.21)$ \\
\hline High LDL-C & 1 (Reference) & & $1.09(0.63-1.87)$ & & $0.96(0.52-1.77)$ \\
\hline Low HDL-C & 1 (Reference) & $\mapsto$ & $0.93(0.74-1.17)$ & ra & $0.97(0.75-1.26)$ \\
\hline High non-HDL-C & 1 (Reference) & $\longmapsto$ & $1.11(0.75-1.63)$ & & $0.85(0.53-1.35)$ \\
\hline Dyslipidemia & 1 (Reference) & $\mapsto-1$ & $0.91(0.76-1.10)$ & $\longmapsto$ & $0.97(0.78-1.20)$ \\
\hline \multirow[t]{2}{*}{ Hyperlipidemia } & 1 (Reference) & $\mapsto \bullet^{\prime}$ & $0.89(0.72-1.10)$ & $\longmapsto$ & $0.99(0.77-1.26)$ \\
\hline & & 1.5 & 2.5 & 0.5 & \\
\hline
\end{tabular}

(b)

Figure 2. Age-specific analysis of the associations between fruit consumption and lipid profile (adjusted for sex, BMI values, residence area, ethnicity, incomes, parental educational attainment, parental weight, vegetable consumption, sugar-sweetened beverages consumption, meat consumption and physical activity) (a) Frequency of fruit consumption; (b) Average daily fruit consumption. TC, total cholesterol; TG, triglycerides; LDL-C, low density lipoprotein-cholesterol; HDL-C, high density lipoprotein-cholesterol; diamond symbol, point estimates; red dashed lines, invalid line. 
When the study sample was restricted according to parental educational attainment, the main associations did not change essentially, but were more significantly pronounced among participants from families with higher parental education levels, compared with those from lower educational families (Supplementary Figure S2). Notably, the results of the two subgroups presented similar according to BMI values (Supplementary Figure S3).

\section{Discussion}

Based on the national representative sample, our findings suggested significant inverse associations between high frequency of fruit consumption per week and moderate daily fruit intake with the odds of adverse lipid profiles. The associations were more pronounced among girls, younger participants and those whose families had a higher educational background, when presented similar by BMI subgroups. These findings supported the health effect of moderate fruit intake frequently to improve the childhood lipid profile.

Our findings were in accordance with previous studies in direction. A case-control study in Jordanians revealed that consumption of banana could reduce the odds of cardiovascular events to about $44 \%$ and $62 \%$ during adulthood when consuming $1-2$ and 3-6 servings per week, respectively [32]. Growing reviews and meta-analyses found consistent associations between fruit consumption and cardiovascular events, diabetes, cancer, and other chronic diseases [33,34]. Among a pediatric population, a diet rich in dietary fiber from fruits was beneficial to a healthy cardiovascular profile, regardless of European children's weight [35]. Since there was evidence supporting that eating behaviors of children and adolescents were likely to persist into adulthood [36], encouraging healthy eating habits among school-aged children might therefore represent an effective primary prevention strategy for reducing the risk of chronic diseases. As recommended by ISPAD Clinical Practice Consensus Guidelines 2018 [37], intake of a variety of fruit should be encouraged among children and adolescents with diabetes, which could be particularly useful in helping to reduce lipid levels.

Although WHO recommends 5 servings ( $400 \mathrm{~g}$ ) of fruits and vegetables per day for the overall population [15], among the pediatric population, studies rarely applied the criteria of 5 servings per day since only few children and adolescents met this recommendation $[16,38]$, thus it was unlikely that any significant association between the recommended levels and lipid health would be detected. Therefore, the absence of a specific frequency and daily amount of fruit consumption for preventing pediatrics' lipid disorders was an important limitation. In the present study, daily fruit consumption of $0.75-1.5$ servings (approximately 150-300 g) for 6-7 days/week was crucial for Chinese pediatrics' lipid health. We speculated that the continued increase in fruit intake would not always promote lipid health, which might be explained by the following reasons: Fructose is a kind of sugar found in fruits, the excess accumulation of which might be positively associated with the level of plasma TG and atherogenic indices [39]. Besides, it could disrupt the utilization of dietary copper, while copper deficiency could produce high TC and high TG [40]. Since there was no consensus regarding the method of assessing fruit consumption in children and adolescents [41], additional studies with high methodological quality are needed to develop informed guidelines for fruit consumption among pediatric population and to better promote lipid health.

However, the exact mechanisms by which increased fruit consumption reduced abnormal lipid profile were not fully understood. As for these inverse associations, antioxidant compounds and polyphenols found in fruit (e.g., vitamin C, carotenoids, and flavonoids) could prevent the oxidation of cholesterol in the arteries [42]. In addition, those compounds could reduce systemic inflammation through cellular signaling processes, therefore preventing atherosclerosis and cardiovascular disease [43]. Apart from this, several components of fruit have cholesterol lowering properties, particularly dietary fiber, as well as high amounts of water and low amounts of saturated fat, which are associated with reduced energy density, hunger control, and satiety [44]. Taken together, the evidence accumulated so far does support that fruit consumption may reduce the odds of lipid disorders. 
Subgroup analyses suggested that the associations between moderate fruit consumption each day and the odds of lipid disorders were more pronounced in girls. A previous study conducted in Korea also emphasized the sex differences, and concluded that low fruit intakes were significantly associated with overweight and obesity among adolescent girls but not in boys [45]. To be noted, we found more frequent of fruit/vegetable consumption and less intake of SSBs among girls; in this context, different behavioral factors may influence the associations in girls and boys. Another potential behavioral explanation was that higher fruit consumption might reflect their health consciousness, consequently, a lower intake of sugar- and fat-rich products, especially in girls.

An interesting age effect was also present; this possibility indicated the potential implications of different lifestyle behaviors in school and preschool children. Our present study indicated these associations were more evident in those aged 5-14 years old. One of the reasons might be that younger children lived healthier lifestyles, while older children and adolescents were under heavier academic pressure, which often led them to overconsume SSBs and meat, as well as to reduce their outdoor activities (Supplementary Table S2). Notably, few studies investigated the association between fruit intakes and lipid profile among different age groups. Therefore we could not discuss an age-specific association between low fruit intakes and adverse lipid profile based on the previous findings.

Targeting the family environment for the promotion of lipid health among children and adolescents is important. Our findings were in agreement with previous surveys which concluded that family education could potentially modify the relationships between dietary behaviors and risks of chronic diseases [30]. It was necessary to influence the fruit consumption preferences of their parents, in order to increase the fruit intake of children [46]. Higher parental educational background may be related to higher household income and thus greater availability of healthy foods, increased nutrition knowledge and higher motivation to follow a healthy lifestyle. In addition, we proposed that health and education programs should consider these findings and be implemented widely to make the public aware of the importance of moderate fruit consumption for the pediatric population. In addition, the similarity of subgroup analysis by BMI showed that the relationship between fruit consumption and lipid profiles was independent of BMI values, to a certain extent. Children and adolescents should consume fruits moderately each day to maintain lipid health, regardless of their weight status.

Our study had strengths of the national representative sample from seven provinces in China, and we focused on 5-19-year-old children and adolescents. However, there were also limitations that should be taken into account when interpreting the findings. Firstly, since $92.56 \%$ of the study population was of Han ethnicity, our results may not be applicable to other ethnic groups. Secondly, we only relied on self-reported dietary intake, which could lead to a certain degree of recall bias. However, in this process, we carried out strict quality control to ensure the reliability. In addition, the dietary data recall of 7 days might not represent long-term dietary behaviors; thus, in future studies, a pilot study using food tracking, such as photo tracking, could be used to accurately assess the frequency and amount of fruit and other dietary intake in individuals. Thirdly, this was a cross-sectional survey and thus could not generate casual relationships of fruit consumption and lipid profile. A randomized controlled trial was desired to confirm our results. Fourth, measuring fruit consumption was complex, with interactions of different fruit compounds, cultural and socioeconomic conditions [47]. Since it was difficult to collect accurate and detailed information on intake of various types of food in a large population survey, we could not identify the specific type of fruits consumed by the participants, and different fruits might have various influences on lipid profiles. However, as for food and diet information, both parent and child questionnaire of children grade 1-3 were reported by parents. In addition, trained project members interpreted all the questionnaires in detail. Appropriate guidance would be given by these project members as effectively as possible. The questionnaires would be rechecked by 3\% within one week for the same participants [18]. Therefore, the quality of diet information was guaranteed largely. Besides, other important dietary 
factors, such as macronutrient and energy intakes, were not available in the present study. In order to minimize possible influence of these factors, we included vegetable, meat and SSBs consumption in the adjusted model. Fifth, the IPAQ-SF questionnaire used in this study was not completely suitable for the assessment of physical activity of children and adolescents. However, due to the open-ended questions of IPAQ-SF, and appropriate guidance would be given by project members, to a certain extent, the IPAQ-SF could reflect the levels of physical activity of children and adolescents. Further studies with more information regarding confounding factors and eating behaviors were desired in the future.

\section{Conclusions}

Our findings confirmed that a diet rich in fruit could be effective for improving lipid health. Given the reason that the basis of dietary intake recommendations is related with its health implications, moderate fruit consumption of approximately 150-300 g each day for 6-7 days/week could be beneficial to lipid health for children and adolescents. Monitoring dietary habits early in childhood and adolescence might have a positive impact on lipid health and overall quality of life. For the pediatric population, indicators of family circumstances should also be applied to identify target groups for interventions aimed at promoting eating habits and lipid health.

Supplementary Materials: The following are available online at https:/ /www.mdpi.com/article/ $10.3390 /$ nu14010063/s1, Figure S1. The size of one serving of fruit or vegetable (as the size of a fist of an ordinary adult). Figure S2: Parental educational attainment-specific analysis of the associations between fruit consumption and lipid profile (adjusted for age, residence area, sex, BMI values, ethnicity, incomes, parental weight, vegetable consumption, sugar-sweetened beverages consumption, meat consumption and physical activity). Figure S3: BMI-specific analysis of the associations between fruit consumption and lipid profile (adjusted for age, residence area, sex, ethnicity, incomes, parental weight, parental educational attainment, vegetable consumption, sugarsweetened beverages consumption, meat consumption and physical activity). Table S1: Prevalence of the abnormal lipid profile $(\mathrm{n}, \%)$. Table S2: Difference of demographics and lifestyle behaviors among age groups.

Author Contributions: Conceptualization, J.L.; Data curation, Y.L., D.G., L.C., M.C. and Y.M.; Formal analysis, J.L. and X.W. (Xinxin Wang); Funding acquisition, Y.D. and J.M.; Methodology, J.L., X.W. (Xinxin Wang), D.G., L.C. and M.C.; Project administration, Y.D. and J.M.; Resources, Z.Z. and J.M.; Software, X.W. (Xinxin Wang) and L.C.; Supervision, Z.Z., X.W. (Xijie Wang), Y.D. and J.M.; Validation, Z.Z., X.W. (Xijie Wang), Y.D. and J.M.; Visualization, Y.L., D.G., L.C., Y.M., Y.Z., J.J., Z.Z., X.W. (Xijie Wang) and Y.D.; Writing — original draft, J.L.; Writing—review and editing, Y.L., X.W. (Xinxin Wang), D.G., M.C., T.M., Q.M., Y.Z., J.J. and X.W. (Xijie Wang). All authors have read and agreed to the published version of the manuscript.

Funding: This research was funded by the Research Special Fund for Public Welfare Industry of Health of the Ministry of Health of China (1147 Project; Grant No. 20122010 to J.M.), China Postdoctoral Science Foundation (BX20200019 and 2020M680266 to Y.D.) and National Natural Science Foundation of China (82103865 to Y.D.).

Institutional Review Board Statement: This study was conducted according to the guidelines laid down in the Declaration of Helsinki and all procedures involving students were approved by the ethics committee of the Peking University (IRB0000105213034).

Informed Consent Statement: All participants and their parents signed informed consents voluntarily.

Data Availability Statement: The data supporting the conclusions of this article will be made available from the corresponding author upon request.

Acknowledgments: The authors would like to acknowledge the support from all the team members and the participating students, teachers, parents and local education and health staff in the programs.

Conflicts of Interest: The authors declare no conflict of interest. 


\section{References}

1. Kit, B.K.; Kuklina, E.; Carroll, M.D.; Ostchega, Y.; Freedman, D.S.; Ogden, C.L. Prevalence of and Trends in Dyslipidemia and Blood Pressure Among US Children and Adolescents, 1999-2012. JAMA Pediatr. 2015, 169, 272-279. [CrossRef]

2. Ding, W.; Cheng, H.; Yan, Y.; Zhao, X.; Chen, F.; Huang, G.; Hou, D.; Mi, J. 10-Year Trends in Serum Lipid Levels and Dyslipidemia Among Children and Adolescents From Several Schools in Beijing, China. J. Epidemiol. 2016, 26, 637-645. [CrossRef] [PubMed]

3. He, H.; Pan, L.; Du, J.; Liu, F.; Jin, Y.; Ma, J.; Wang, L.; Jia, P.; Hu, Z.; Shan, G. Prevalence of, and biochemical and anthropometric risk factors for, dyslipidemia in children and adolescents aged 7 to 18 years in China: A cross-sectional study. Am. J. Hum. Biol. 2019, 31, e23286. [CrossRef] [PubMed]

4. Ding, W.; Dong, H.; Mi, J. Prevalence of dyslipidemia in Chinese children and adolescents:a Meta-analysis. Zhonghua Liu Xing Bing Xue Za Zhi = Zhonghua Liuxingbingxue Zazhi 2015, 36, 71-77. [PubMed]

5. Lozano, P.; Henrikson, N.; Morrison, C.; Dunn, J.; Nguyen, M.; Blasi, P.; Whitlock, E. Lipid Screening in Childhood and Adolescence for Detection of Multifactorial Dyslipidemia: Evidence Report and Systematic Review for the US Preventive Services Task Force. JAMA Pediatr. 2016, 316, 634-644. [CrossRef]

6. Rauber, F.; Campagnolo, P.; Hoffman, D.; Vitolo, M. Consumption of ultra-processed food products and its effects on children's lipid profiles: A longitudinal study. Nutr. Metab. Cardiovasc. Dis. 2015, 25, 116-122. [CrossRef]

7. Lim, S.S.; Vos, T.; Flaxman, A.D.; Danaei, G.; Shibuya, K.; Adair-Rohani, H.; AlMazroa, M.A.; Amann, M.; Anderson, H.R.; Andrews, K.G.; et al. A comparative risk assessment of burden of disease and injury attributable to 67 risk factors and risk factor clusters in 21 regions, 1990-2010: A systematic analysis for the Global Burden of Disease Study 2010. Lancet 2012, 380, $2224-2260$. [CrossRef]

8. Van Duyn, M.; Pivonka, E. Overview of the health benefits of fruit and vegetable consumption for the dietetics professional: Selected literature. J. Am. Diet. Assoc. 2000, 100, 1511-1521. [CrossRef]

9. LeDoux, T.A.; Hingle, M.D.; Baranowski, T. Relationship of fruit and vegetable intake with adiposity: A systematic review. Obes. Rev. 2010, 12, e143-e150. [CrossRef]

10. Pan, Y.; Pratt, C. Metabolic syndrome and its association with diet and physical activity in US adolescents. J. Am. Diet. Assoc. 2008, 108, 276-286. [CrossRef]

11. Rosi, A.; Paolella, G.; Biasini, B.; Scazzina, F. SINU Working Group on Nutritional Surveillance in Adolescents. Dietary habits of adolescents living in North America, Europe or Oceania: A review on fruit, vegetable and legume consumption, sodium intake, and adherence to the Mediterranean Diet. Nutr. Metab. Cardiovasc. Dis. 2019, 29, 544-560. [CrossRef]

12. John, J.; Ziebland, S.; Yudkin, P.; Roe, L.; Neil, H. Effects of fruit and vegetable consumption on plasma antioxidant concentrations and blood pressure: A randomised controlled trial. Lancet 2002, 359, 1969-1974. [CrossRef]

13. Keung, V.; Lo, K.; Cheung, C.; Tam, W.; Lee, A. Changes in dietary habits and prevalence of cardiovascular risk factors among school students in Macao, China. Obes. Res. Clin. Pract. 2019, 13, 541-547. [CrossRef] [PubMed]

14. Cardoso Chaves, O.; Franceschini, S.C.; Machado Rocha Ribeiro, S.; Ferreira Rocha Sant Ana, L.; Garçon De Faria, C.; Priore, S.E. Anthropometric and biochemical parameters in adolescents and their relationship with eating habits and household food availability. Nutr. Hosp. 2013, 28, 1352-1356. [PubMed]

15. World Health Organization. Diet, Nutrition, and the Prevention of Chronic Diseases. Report of the Joint WHO/FAO Expert Consultation; (WHO Technical Report Series, no. 916); World Health Organization: Geneva, Switzerland, 2002.

16. Collese, T.S.; Nascimento-Ferreira, M.V.; De Moraes, A.C.F.; Rendo-Urteaga, T.; Bel-Serrat, S.; Moreno, L.A.; Carvalho, H.B. Role of fruits and vegetables in adolescent cardiovascular health: A systematic review. Nutr. Rev. 2017, 75, 339-349. [CrossRef] [PubMed]

17. Sharma, S.P.; Chung, H.J.; Kim, H.J.; Hong, S.T. Paradoxical Effects of Fruit on Obesity. Nutrients 2016, 8, 633. [CrossRef]

18. Chen, Y.; Ma, L.; Ma, Y.; Wang, H.; Luo, J.; Zhang, X.; Luo, C.; Wang, H.; Zhao, H.; Pan, D.; et al. A national school-based health lifestyles interventions among Chinese children and adolescents against obesity: Rationale, design and methodology of a randomized controlled trial in China. BMC Public Health 2015, 15, 210. [CrossRef]

19. Dong, Y.H.; Zou, Z.Y.; Yang, Z.P.; Wang, Z.H.; Jing, J.; Luo, J.Y.; Zhang, X.; Luo, C.Y.; Wang, H.; Zhao, H.P.; et al. Association between high birth weight and hypertension in children and adolescents: A cross-sectional study in China. J. Hum. Hypertens. 2017, 31, 737-743. [CrossRef]

20. Gui, Z.-H.; Zhu, Y.-N.; Cai, L.; Sun, F.-H.; Ma, Y.-H.; Jing, J.; Chen, Y.-J. Sugar-Sweetened Beverage Consumption and Risks of Obesity and Hypertension in Chinese Children and Adolescents: A National Cross-Sectional Analysis. Nutrients 2017, 9 , 1302. [CrossRef] [PubMed]

21. Yang, Y.; Dong, B.; Zou, Z.; Wang, S.; Dong, Y.; Wang, Z.; Ma, J. Association between Vegetable Consumption and Blood Pressure, Stratified by BMI, among Chinese Adolescents Aged 13-17 Years: A National Cross-Sectional Study. Nutrients 2018, 10, 451. [CrossRef]

22. Ho, S.-Y.; Wong, B.Y.-M.; Lo, W.-S.; Mak, K.-K.; Thomas, G.N.; Lam, T.H. Neighbourhood food environment and dietary intakes in adolescents: Sex and perceived family affluence as moderators. Int. J. Pediatr. Obes. 2010, 5, 420-427. [CrossRef]

23. Hicks, T.M.; Knowles, S.O.; Farouk, M. Global Provisioning of Red Meat for Flexitarian Diets. Front. Nutr. 2018, 5, 50. [CrossRef] [PubMed]

24. The Chinese Nutrition Society. Dietary Guidelines for Chinese School-Age Children (2016); People's Medical Publishing House: Beijing, China, 2016. 
25. Lee, P.H.; Macfarlane, D.J.; Lam, T.H.; Stewart, S.M. Validity of the international physical activity questionnaire short form (IPAQ-SF): A systematic review. Int. J. Behav. Nutr. Phys. Act. 2011, 8, 115. [CrossRef] [PubMed]

26. Craig, C.L.; Marshall, A.L.; Sjöström, M.; Bauman, A.E.; Booth, M.L.; Ainsworth, B.E.; Pratt, M.; Ekelund, U.; Yngve, A.; Sallis, J.F.; et al. International Physical Activity Questionnaire: 12-Country Reliability and Validity. Med. Sci. Sports Exerc. 2003, 35, 1381-1395. [CrossRef]

27. Zhou, B.-F. Effect of body mass index on all-cause mortality and incidence of cardiovascular diseases-report for meta-analysis of prospective studies open optimal cut-off points of body mass index in Chinese adults. Biomed. Environ. Sci. 2002, 15, 245-252. [PubMed]

28. Expert Panel on Integrated Guidelines for Cardiovascular Health and Risk Reduction in Children and Adolescents; National Heart, Lung, and Blood Institute. Expert panel on integrated guidelines for cardiovascular health and risk reduction in children and adolescents: Summary report. Pediatrics 2011, 128 (Suppl. 5), S213-S256. [CrossRef]

29. Group of China Obesity Task Force. Body mass index reference norm for screening overweight and obesity in Chinese children and adolescents. Zhonghua Liu Xing Bing Xue Za Zhi 2004, 25, 97-102.

30. Spinneker, A.; Egert, S.; González-Gross, M.; Breidenassel, C.; Albers, U.; Stoffel-Wagner, B.; Huybrechts, I.; Manios, Y.; Venneria, E.; Molnar, D.; et al. Lipid, lipoprotein and apolipoprotein profiles in European adolescents and its associations with gender, biological maturity and body fat-The HELENA Study. Eur. J. Clin. Nutr. 2012, 66, 727-735. [CrossRef]

31. Kocaoglu, B.; Manios, Y.; Dimitriou, M.; Kolotourou, M.; Keskin, Y.; Sur, H.; Hayran, O.; Manios, Y. Parental educational level and cardiovascular disease risk factors in schoolchildren in large urban areas of Turkey: Directions for public health policy. BMC Public Health 2005, 5, 13. [CrossRef]

32. Tayyem, R.F.; Al-Bakheit, A.; Hammad, S.S.; Al-Shudifat, A.-E.; Azab, M.; Bawadi, H. Fruit and vegetable consumption and cardiovascular diseases among Jordanians: A case-control study. Central Eur. J. Public Health 2020, 28, 208-218. [CrossRef]

33. Wu, Y.; Zhang, D.; Jiang, X.; Jiang, W. Fruit and vegetable consumption and risk of type 2 diabetes mellitus: A dose-response meta-analysis of prospective cohort studies. Nutr. Metab. Cardiovasc. Dis. 2015, 25, 140-147. [CrossRef] [PubMed]

34. Wang, X.; Ouyang, Y.; Liu, J.; Zhu, M.; Zhao, G.; Bao, W.; Hu, F.B. Fruit and vegetable consumption and mortality from all causes, cardiovascular disease, and cancer: Systematic review and dose-response meta-analysis of prospective cohort studies. BMJ 2014, 349, g4490. [CrossRef] [PubMed]

35. Larrosa, S.; Luque, V.; Grote, V.; Closa-Monasterolo, R.; Ferré, N.; Koletzko, B.; Verduci, E.; Gruszfeld, D.; Xhonneux, A.; Escribano, J. Fibre Intake Is Associated with Cardiovascular Health in European Children. Nutrients 2020, 13, 12. [CrossRef] [PubMed]

36. Craigie, A.M.; Lake, A.; Kelly, S.; Adamson, A.; Mathers, J.C. Tracking of obesity-related behaviours from childhood to adulthood: A systematic review. Maturitas 2011, 70, 266-284. [CrossRef]

37. Smart, C.E.; Annan, F.; Higgins, L.A.; Jelleryd, E.; Lopez, M.; Acerini, C.L. ISPAD Clinical Practice Consensus Guidelines 2018 Nutritional management in children and adolescents with diabetes. Pediatr. Diabetes 2018, 19 (Suppl. S27), 136-154. [CrossRef]

38. Panagiotopoulos, C.; Nguyen, D.; Smith, J. Cardiovascular risk factors and health behaviours in elementary school-age Inuvialuit and Gwich'in children. Paediatr. Child Health 2014, 19, 256-260. [CrossRef]

39. Czerwonogrodzka-Senczyna, A.; Rumińska, M.; Majcher, A.; Credo, D.; Jeznach-Steinhagen, A.; Pyrżak, B. Fructose Consumption and Lipid Metabolism in Obese Children and Adolescents. Adv. Exp. Med. Biol. 2019, 1153, 91-100. [CrossRef]

40. Klevay, L.M. Metabolic interactions among dietary cholesterol, copper, and fructose. Am. J. Physiol. Endocrinol. Metab. 2010, 298, E138-E139. [CrossRef]

41. Collins, C.E.; Watson, J.; Burrows, T. Measuring dietary intake in children and adolescents in the context of overweight and obesity. Int. J. Obes. 2010, 34, 1103-1115. [CrossRef]

42. Asplund, K. Antioxidant vitamins in the prevention of cardiovascular disease: A systematic review. J. Intern. Med. 2002, 251, 372-392. [CrossRef]

43. Joseph, S.V.; Edirisinghe, I.; Burton-Freeman, B.M. Fruit Polyphenols: A Review of Anti-inflammatory Effects in Humans. Crit. Rev. Food Sci. Nutr. 2016, 56, 419-444. [CrossRef] [PubMed]

44. Rolls, B.J.; Ello-Martin, J.A.; Tohill, B.C. What can intervention studies tell us about the relationship between fruit and vegetable consumption and weight management? Nutr. Rev. 2004, 62, 1-17. [CrossRef] [PubMed]

45. You, J.; Choo, J. Adolescent Overweight and Obesity: Links to Socioeconomic Status and Fruit and Vegetable Intakes. Int. J. Environ. Res. Public Health 2016, 13, 307. [CrossRef] [PubMed]

46. Groele, B.; Głąskka, D.; Gutkowska, K.; Guzek, D. Mother's Fruit Preferences and Consumption Support Similar Attitudes and Behaviors in Their Children. Int. J. Environ. Res. Public Health 2018, 15, 2833. [CrossRef]

47. Corella, D.; Ordovás, J. Biomarkers: Background, classification and guidelines for applications in nutritional epidemiology. Nutr. Hosp. 2015, 31 (Suppl. S3), 177-188. [PubMed] 